\title{
Tailoring the Molecular Skeleton of Aza-BODIPYs to Design Photostable Red-Light-Emitting Laser Dyes
}

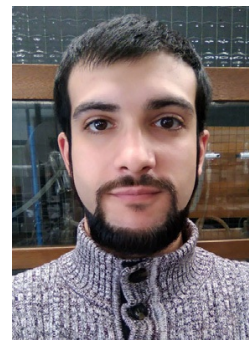

A. Prieto-Castañeda

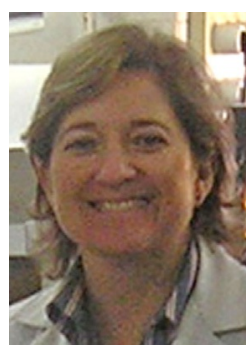

A. R. Agarrabeitia

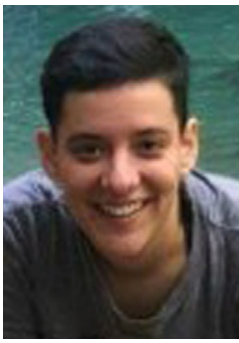

E. Avellanal-Zaballa

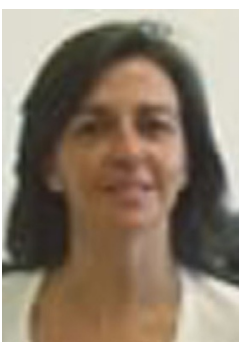

I. García-Moreno

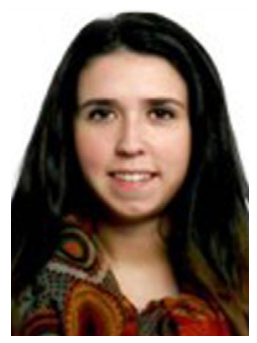

L. Gartzia-Rivero

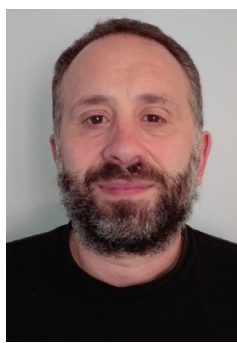

J. Bañuelos

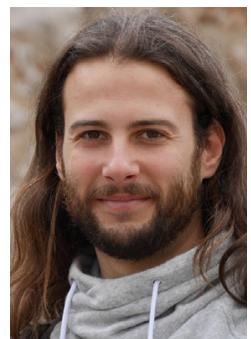

L. Cerdán

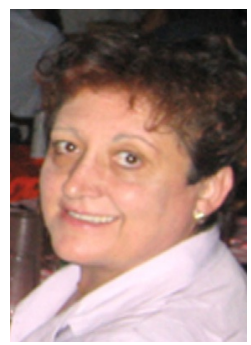

M. J. Ortiz
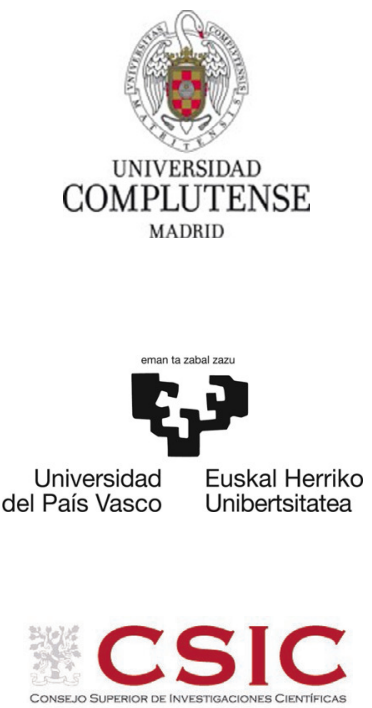

The front cover artwork is provided by Maria Cristina Devesa, the Organic Chemistry Group from the Universidad Complutense de Madrid (UCM), the Molecular Spectroscopy Group from Universidad del País Vasco (UPV/EHU) and the Laser Materials Group from Instituto Rocasolano (CSIC). The image shows the bright and stable deep-red emission from the designed aza-BODIPY chromophores. Such laser emission can be pushed towards the near-infrared upon a rational selection of the substitution pattern of the fluorophore. Read the full text of the Article at 10.1002/cptc.201800225.

What prompted you to investigate this topic?

The development of organic fluorophores able to display emission in the red edge of the visible or the near-infrared region is a long sought after challenge. This kind of radiation features advantages for telecommunications and biomedicine owing to its lower interference with the environment and deeper penetration into tissues.

\section{How did the collaboration on this project start?}

We have been working actively in the field of dye chemistry, mainly with BODIPYs. We aimed to apply the accumulated knowledge about the synthesis and photophysical signatures of BODIPYs to develop long-wavelength-emitting dyes with improved photonic behaviour.

What was the inspiration for this cover design? We aimed to highlight the efficient and long-lasting laser emission of the aza-BODIPYs and the modulation of this signal, from the red to the near-infrared region, by means of a rational design of the molecular structure.

\section{What is the most significant result of this study?}

The designed experimental set-up featuring an optical parametric oscillator pumping with a Nd:YAG laser allows us to demonstrate for the first time the suitability of aza-BODIPYs as active media for stable red-emitting tunable lasers.
Acknowledgments

This work was supported by the Spanish Ministerio de Economia y Competitividad (MAT2017-83856-C3) and Gobierno Vasco (IT912-16).

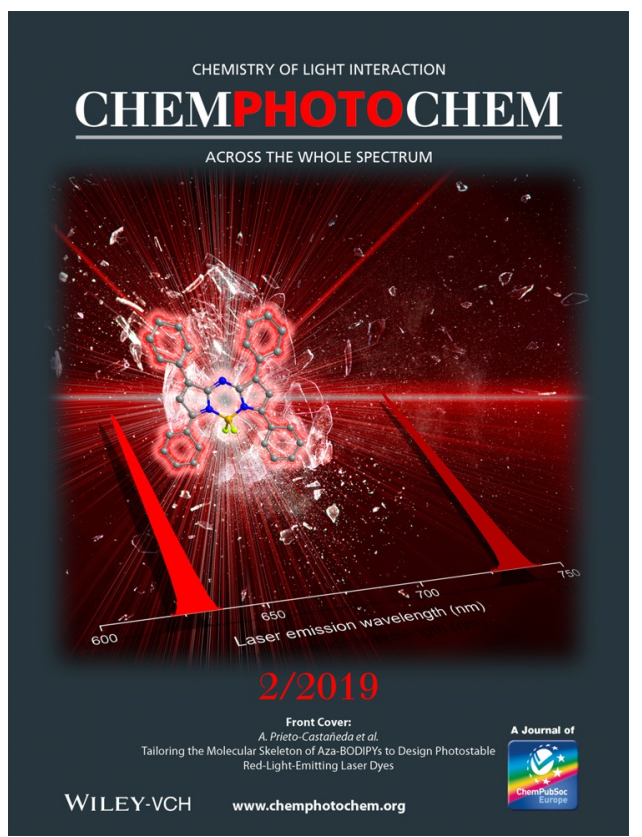

